\title{
RESTRIÇÕES DE CONTROLE SOBRE CORES DECOMPOSTAS: UMA PROPOSTA NO CONTROLE SUPERVISÓRIO DE SISTEMAS A EVENTOS DISCRETOS UTILIZANDO REDES DE PETRI COLORIDAS
}

\author{
F. A. de Alencar Menezes* \\ alencarmenezes@gmail.com \\ Bruno de A. Prata
baprata ${ }^{\ddagger}$ ifce. edu. br \\ Giovanni C. Barroso ${ }^{\dagger}$ \\ gcbefisica.ufc.br \\ *UEVA - Universidade Estadual Vale do Acaraú, Sobral, Ce, Brasil \\ †UFC - Universidade Federal do Ceará, Fortaleza, Ce, Brasil \\ ${ }^{\ddagger}$ IFCE - Instituto Federal de Educação, Ciência e Tecnologia, Quixadá, Ce, Brasil
}

\begin{abstract}
Control Restrictions on Decomposed Colors: An Approach for Supervisory Control of Discrete Event Systems Using Colored Petri Nets

This paper presents the mathematical proof of the method for the synthesis of supervisors called Constraint of Control on Decomposed Colors, which is based on concepts of Supervisory Control Theory, using as main tool the coloured Petri nets to model systems discrete event, in order to find supervisors that meet those systems subject to constraints. By extension, it is proposed a method to reduce the number of controllers called Fusion of Controllers.
\end{abstract}

KEYWORDS: Supervisory control, coloured Petri nets, discrete event systems.

\section{RESUMO}

Este trabalho apresenta a prova matemática do método para a síntese de supervisores denominado Restrição de Controle sobre Cores Decompostas, o qual é baseado nos conceitos da Teoria de Controle Supervisório, utilizando como principal ferramenta as redes de Petri coloridas para modelar sistemas

\footnotetext{
Artigo submetido em 03/11/2010 (Id.: 1213)

Revisado em 04/04/2011, 13/09/2011

Aceito sob recomendação do Editor Associado Prof. Vitor Ferreira Romano
}

de eventos discretos, a fim de encontrar supervisores que satisfaçam esses sistemas sujeitos a restrições. Por extensão, é proposto um método para reduzir o número de controladores denominado Fusão de Controladores.

PALAVRAS-CHAVE: Controle supervisório, rede de Petri colorida, sistemas a eventos discretos.

\section{INTRODUÇÃO}

A teoria de controle supervisório (TCS) desenvolve-se a partir de (Ramadge and Wonham, 1987) e (Ramadge and Wonham, 1989) e surge da necessidade de uma ferramenta sistemática (Hopcroft et al., 2006) para especificação e implementação do controle dos sistemas a eventos discretos (SED). Nessa teoria um SED é modelado por um autômato cujo comportamento é descrito por linguagens formais geradas do mesmo. O problema de controle resume-se em encontrar o supervisor, tal que, associado ao sistema, gere a linguagem especificada. Três fundamentos compõem a TCS: controlabilidade de uma linguagem, existência do supervisor e existência de máxima linguagem controlável. Entretanto, alguns inconvenientes são evidenciados ao se usar autômatos de estado como ferramenta para modelagem de SED, tais como o crescimento exponencial do número de estados quando o sistema é de grande porte e a pouca visibilidade 
gráfica que limita seu uso a sistemas de menor tamanho. Diante disso, propõe-se uma abordagem da TCS utilizando como ferramenta as redes de Petri (RP), onde se ganha no recurso da visualização gráfica e na síntese de supervisores. Dessa forma, ao nível da linguagem formal baseada em autômatos, a relação do estudo de linguagens da TCS com rede de Petri permite caracterizar a classe de problemas de controle que podem ser resolvidos através de modelos em rede de Petri, ou seja, a classe dos problemas para os quais um supervisor em rede de Petri existe. Nesse contexto, as redes de Petri coloridas (RPC), como uma ferramenta poderosa para modelagem de SED através de funções associadas aos arcos, oferecem a condição para modelagem de sistemas de grande porte sem prejuízo de visualização gráfica e maior recurso de análise. Nesse trabalho, no contexto dos SED, é estudada uma estrutura modelada em RPC tendo como referência a TCS no projeto de um supervisor. Assim, são usadas técnicas de análise em rede de Petri a fim de provar que o supervisor temas propriedades desejadas.

Como objeto central do artigo, apresenta-se a comprovação do método denominado Restrições de Controle sobre Cores Decompostas (RCCD), de controle de SED utilizando RPC, proposto por (Prata et al., 2008) com a prova do Teorema 2, uma extensão do Teorema 1 de (Moody and Antsaklis, 1998). Como extensão ao Teorema 2, apresenta-se o Teorema 3, como um método para redução do número de controladores desses supervisores denominado Fusão de Controladores.

A estrutura do trabalho compreende o esboço da TCS com autômatos e uma revisão da literatura da TCS utilizando RP, na Seção 2; a formalização do método RCCD com contextos de aplicações na Seção 3; o método Fusão de Controladores apresentado na Seção 4 e a conclusão na Seção 5.

\section{O CONTROLE SUPERVISÓRIO}

O problema do controle supervisório é de um modo geral resolvido pela TCS, que separa o sistema a ser controlado (open loop dynamics) do controlador (feedback control) e envolve a modelagem, especificação do comportamento e síntese do supervisor. No processo, a síntese de um supervisor é realizada para um dado modelo com o objetivo de satisfazer uma especificação de comportamento desejada. Dessa forma, o supervisor é um agente externo que possui habilidade de observar os eventos gerados pelo sistema e influenciar no seu comportamento através de entradas de controle. Em malha fechada, a ação de controle do supervisor garante que o funcionamento do sistema esteja de acordo com uma especificação dada.

Na TCS, segundo (Barkaoui et al., 1997) e (Cury, 2001), um $S E D$ é modelado por um par de linguagens, $L$ e $L_{m}$, bem definidas, mais o conjunto de eventos $\Sigma$. $L$ representa o conjunto de todas as sequências de eventos que o sistema pode gerar a partir de seu estado inicial. $L$ é fechada, ou seja $L=\bar{L}=\left\{s \in \Sigma^{*}:\left(\exists t \in \Sigma^{*}\right)\right.$ st $\left.\in L\right\}$ e $L_{m} \subseteq L$ é a linguagem marcada que representa as tarefas completadas do sistema. Seja

$$
G=\left(Q, \Sigma, \delta, \Sigma_{G} \cdot q_{0} Q_{m}\right)
$$

o gerador associado a estas duas linguagens. Por definição, $\Sigma^{*}$ representa o conjunto de todas as sequências finitas em $\Sigma$, incluindo a sequência nula (ou vazia) $\epsilon$; $Q$ é o conjunto de estados de $G ; \delta: \Sigma \times Q \rightarrow Q$ é a função de transição de estados, tal que, $\delta(\epsilon, q)=q$ e $\delta(\sigma, q)=q^{\prime}$ onde $q, q^{\prime} \in Q$ e $\sigma \in \Sigma ; q_{0} \in Q$ é estado inicial de $G$ e $Q_{m} \subseteq Q$ é o conjunto de estados marcados. $\Sigma_{G}: Q \rightarrow 2^{\Sigma}$ denota o conjunto ativo de eventos $q \in Q$ num estado, tal que, $\delta(q, s)$ é definida. Segue que

$$
\begin{aligned}
L & =\mathcal{L}(G)=\left\{s \in \Sigma^{*}: \delta\left(q_{0}, s\right) \text { é definida }\right\} \\
L_{m} & =\mathcal{L}_{m}(G)=\left\{s \in \mathcal{L}(G): \delta\left(q_{0}, s\right) \in Q_{m}\right\},
\end{aligned}
$$

$G$ é dito não-bloqueante (trim) quando

$$
\mathcal{L}(G)=\overline{\mathcal{L}_{m}(G)},
$$

caso contrário é dito bloqueante. Uma situação a ser considerada é que $G$ pode alcançar um estado em que $\Sigma_{G}(q)=\phi$, com $q \notin Q_{m}$. Nesse caso, esse um estado de impasse em que nenhum outro evento pode ser executado como sequncia. Tal estado denominado deadlock. Outro estado de impasse quando $G$ entra em um ciclo de estados sem terminar a tarefa especificada ou quando ele entra em um ciclo de estados no marcados sem transio para sair do ciclo. Tal ciclo de estados denominado livelock. Particionando $\Sigma$ em dois subconjuntos disjuntos obtm-se: $\Sigma_{c}$, o subconjunto formado pelos eventos controlveis do sistema (eventos que podem ser impedidos de acontecer, atravs de controle) e $\Sigma_{u}$ o subconjunto formado pelos eventos no controlveis do sistema (eventos que no podem ser desativados por controle). Quando o autmato $G$ tem um "comportamento proibido" (gera tarefa no especificada), o objetivo do controle ento restringir o comportamento do sistema no controlado, descrito por $G$, dentro dos limites do "comportamento no-proibido", dado como um subconjunto de $\mathcal{L}(G)$ sse controle pode ser alcanado conectando o sistema em malha com um supervisor $S$ (um controlador externo), sob a restrio que $S$ nunca desabilitar um evento no controlvel. ( $S \quad \Sigma_{u}$ - habilitado). O sistema resultante 
em malha fechada denotado por $S / G$, a linguagem gerada $\mathcal{L}(S / G) \subseteq \mathcal{L}(G)$ e sua linguagem marcada consistem exatamente das sequncias marcadas de $G$ que esto sob o controle de $S$, tal que, $\mathcal{L}_{m}(S / G)=\mathcal{L}(S / G) \cap \mathcal{L}_{m}(G)$. Em problemas onde o bloqueio motivo de preocupao, o "comportamento no-proibido" dado como um subconjunto de $\mathcal{L}_{m}(G)$. Assim, uma propriedade importante que $S$ deve satisfazer ser um supervisor prprio, dessa forma, o sistema sob superviso $S / G$ no-bloqueante, ou seja,

$$
\mathcal{L}(S / G)=\overline{\mathcal{L}_{m}(S / G)}
$$

Uma vez que as sequncias marcadas representam as tarefas concludas ou registram a realizao de alguma operao em particular (por escolha de modelagem), o bloqueio significa que o sistema controlado no pode terminar a execuo das tarefas desejveis.

Dada uma linguagem $K \subseteq \mathcal{L}_{m}(G)$ existe um supervisor $S$ no-bloqueante para $G$, tal que, $\mathcal{L}_{m}(S / G)=K$ se e somente se, $K$ for controlvel. Seja $C(E)$ o conjunto de linguagens controlveis em $K \subseteq E \subseteq \Sigma^{*}$ e $A \subseteq \mathcal{L}_{m}(S / G) \subseteq E$, o problema do controle supervisrio possui soluo se e somente se, $C(E)$ possui um elemento supremo sup $C(E) \supseteq A$. Nessas condies, sup $C(E)$ representa o comportamento menos restritivo possvel para se implantar no sistema $G$, satisfazendo as especificaes $A$ e $E$. Assim, $S$ timo, tal que,

$$
\mathcal{L}_{m}(S / G)=\sup C(E)
$$

\subsection{Comentários sobre Abordagens da TCS com Rede de Petri}

usual modelar e controlar um SED por intermdio de um autmato finito (Ramadge and Wonham, 1989), porm, tal abordagem extremamente laboriosa e torna-se impraticvel para sistemas com grande nmero de estados e eventos. Um modo de contornar este problema modelar e controlar um SED por intermdio das RP. Conforme (Murata, 1989), as RP so um tipo de grafo bipartido, dirigido e ponderado, que consegue captar a dinmica de um Sistema a Eventos Discretos, estas propiciam uma representao compacta de um sistema por no representarem explicitamente todo o espao de estados do sistema modelado. A representao grfica intuitiva, bem como sua poderosa formulao algbrica, tornam as RP uma das ferramentas mais indicadas para o controle supervisrio de SED (Moody and Antsaklis, 1998) e (Iordache and Antsaklis, 2006).

Segundo (Lima and Oliveira, 2007), os supervisores usando RP apresentam alguns problemas, dentre os quais: Supervisão Ótima Comprometida, em que a explosão do grafo de alcançabilidade de estados é um fator que dificulta a imposição de controladores em uma RP. Redes com poucos lugares e transições podem atingir uma imensa quantidade de estados. Numa RPC, à medida que o número de cores aumenta o número de combinações entre as cores que podem compor a marcação de um dado lugar também, de modo que o grafo de alcançabilidade cresce exponencialmente (Jensen and Kristensen, 2009). Assim, ao se evitar os algoritmos de análise de alcançabilidade, devido à complexidade computacional, perde-se a garantia de uma supervisão ótima, ou seja, aquela em que a ação do controlador é minimamente restritiva (onde a ação de controle só ocorre para evitar que os requisitos exigidos para o funcionamento do sistema sejam contrariados). Uma forma de garantir uma supervisão ótima tem sido restringir o método a uma classe de sistema que podem ser modelados pelas chamadas RP determinísticas, ou seja, aquelas em que duas ou mais transições associadas a um mesmo evento, não podem estar habilitadas ao mesmo tempo (Cassandras and Lafortune, 2008). Bloqueio, em que, diferentemente da TCS baseada em autômatos, os métodos baseados em RP, para síntese de controladores, não garantem que os controladores obtidos sejam não-bloqueantes. Nesse caso, para garantir que o sistema controlado não tenha bloqueio, é preciso ter algumas considerações estruturais da rede, das condições iniciais e das transições não controláveis, ou seja, a RP em questão tem que ser "viva". Uma RP é dita viva se for possível executar todas as suas ações a partir de qualquer uma das marcações alcançáveis da rede. Esta propriedade é muito importante na análise de sistemas digitais, uma vez que, quando verificada, indica a inexistência de bloqueios no sistema modelado. Porém, a verificação da vivacidade irrestrita de uma RP é, freqüentemente, muito cara. Por esta razão é possível relaxar esta propriedade, adotando-se níveis de vivacidade a fim de se satisfazer níveis de necessidades de modelagem sem bloqueio. Conflito, onde ocorre quando vários controladores agem sobre um mesmo sistema é necessário garantir que esta ação conjunta não seja conflitante, ou seja, a ação de um controlador não leve à inibição de outro controlador.

\subsubsection{Métodos destacados na literatura de síntese de supervisores utilizando $\mathbf{R P}$}

As RP controladas permitem a concepção de um modelo geral, que pode ser facilmente manuseado através de especificações impostas pela adição de lugares de controle. A marcação dos lugares de controle destina-se a controlar o sistema modelado, com vistas a restringir o grafo de alcançabilidade da rede às especificações impostas. As RP controladas possuem dois tipos de lugares distintos: os lugares de estado e os lugares de controle. O método consiste em designar lugares de controle de modo a regular o processo de disparo das transições de acordo com especificações funcionais. Os lugares 
de estado, usualmente, possuem um significado físico (máquina em operação, peça sendo processada, localidade geográfica, etc.). A existência de uma ficha em um lugar de estado significa que a condição associada àquele lugar, naquele instante, é satisfeita. Já os lugares de controle representam regras de monitoramento do sistema, podendo, ou não, ter um significado bem definido. Os lugares de controle são um conjunto de condições externas que influenciam na progressão das marcações em uma rede (Holloway et al., 1997).

\section{Método das restrições generalizadas mutuamente excludentes}

Em SED, em diversas circunstâncias, processos distintos compartilham, concorrentemente, recursos. Restrições de exclusão mútua permitem que tais recursos sejam compartilhados pelos processos do sistema em análise. (Giua, 1992) propôs um método, denominado Restrições Generalizadas Mutuamente Excludentes, que impõe restrições de controle em redes de Petri lugar-transição através da síntese de monitores. Nesse caso, os monitores são lugares de estado idênticos aos lugares de uma rede de Petri ordinária, e não os lugares de controle das redes de Petri controladas e quando são adicionados à estrutura da rede, o monitor modifica o comportamento do sistema de modo a evitar o alcance de marcações que não satisfaçam às restrições. Em redes com transições não controláveis, o controle torna-se um problema mais geral de restrição de marcações proibidas. Contudo, para redes vivas e conservativas, ainda que haja transições não controláveis na rede que modela um SED não controlado, monitores podem ser impostos.

\section{Método das redes de Petri com funções de habilita- ção de transições}

O procedimento para síntese de supervisores de SED via rede de Petri com funções de habilitação de transições (RPFHT) inicia com a concepção do modelo do sistema, em sua forma não controlada. A seguir, deve-se construir a árvore de cobertura de estados do modelo de modo a avaliar o conjunto de marcações alcançáveis pela rede. As especificações de controle são apontadas para, em seguida, serem obtidas as especificações possíveis. As funções de habilitação das transições são determinadas e, por fim, têm-se a RPFHT supervisora. Nesse método, a RPFHT pode ser transformada numa RP ordinária equivalente. Para tanto, basta que as funções de habilitação de transições sejam substituídas por arcos ponderados, que ligam as transições que contêm funções de habilitação aos lugares da rede, de modo que as restrições impostas ao disparo das transições não sejam alteradas (Barroso, 1996) e (Barroso et al., 1996).

\section{Método dos invariantes de lugar}

Dada uma RP com $n$ lugares e m transições, o objetivo do controle é forçar os processos a obedecerem a restrições, tal que, $l_{1} M\left(p_{i}\right)+l_{2} M\left(p_{j}\right) \leq b_{1}$, onde $l_{1}, l_{2}$ e $b_{1}$ so inteiros. Essas restrições são equivalentes a um conjunto de restrições generalizadas mutuamente excludentes. Nesse caso, com o auxílio de uma variável de folga $M_{s}>0$, pode-se ter $l_{1} M\left(p_{i}\right)+l_{2} M\left(p_{j}\right)+M_{s}=b_{1}$. A variável de folga representará a adição de um lugar de controle cuja marcação irá satisfazer a condição de igualdade. Esse argumento torna-se suficiente para a síntese do supervisor segundo (Moody and Antsaklis, 1998).

\section{Outras abordagens no contexto das RP}

(Holloway and Krogh, 1990) comparam a abordagem por invariantes de lugar com o tipo de restrição mais genérica que pode ser definida sobre o conjunto de marcações de um sistema. Foi observado que nem sempre existe um invariante de lugar equivalente a uma restrição de marcações proibidas. Yamalidou et al. 1996 utilizam invariantes de lugar para calcular, através de multiplicação de matrizes, um controlador por realimentação de estados. Este método de controle pode ser aplicado a sistemas cujas restrições são expressas como inequações algébricas ou expressões lógicas que contêm elementos dos vetores de marcação e/ou disparo. (Moody et al., 1996) generalizam o conceito de invariantes de lugar a RP que possuem restrições não controláveis e/ou não observáveis, mas não garantem a máxima permissividade para o caso geral. Nessa metodologia não devem existir arcos conectando os lugares de controle às transições não controláveis, ao mesmo tempo em que não devem existir arcos das transições não observáveis aos lugares de controle. (Braga, 2006) conclui que a utilização de RP propõe uma abordagem baseada em princípios de verificação formal para a supervisão de SED, cuja metodologia faz uso da Teoria das Regiões e das equações $\mu$-calculus, generalizando a abordagem de (Ramadge and Wonham, 1987) e (Ramadge and Wonham, 1989).

\section{Abordagens no contexto das RPC}

(Makungu et al., 1999) apresentam uma contribuição no sentido de reduzir a complexidade de problemas de controle de SED, através da proposição de um algoritmo, dedicado às RPC com número finito de cores e k-limitadas. Ao contrário de algoritmos convencionais que exploram todo o conjunto de estados alcançáveis, este evita uma busca exaustiva do espaço de estados, explorando uma especificação de simetria. Tal algoritmo oferece resposta satisfatória quando aplicado a grandes processos, mas estruturado com componentes similares. Além disso, esta abordagem leva a uma representa- 
ção de supervisores, que são menores que os obtidos com as abordagens baseadas em autômato. (Prata et al., 2008) apresentam um novo método denominado Restrições de Controle sobre Cores Decompostas (RCCD) onde é possível separar as cores da rede de forma que cada função dos arcos possa ser expressa como um valor inteiro. Desse modo, pode-se analisar, separadamente, a matriz de incidência relativa a cada cor, a fim de definir controladores segundo (Moody and Antsaklis, 1998), referência principal para esse trabalho.

\section{RCCD - RESTRIÇÕES DE CONTROLE SOBRE CORES DECOMPOSTAS}

Como formalismo preliminar para esse trabalho, o método dos invariantes de lugar para síntese de supervisores utilizando RP de (Moody and Antsaklis, 1998), descrito no Teorema 1, é ponto de partida para a formalização matemática do método RCCD. Os invariantes de lugar são uma das propriedades estruturais das RP que dependem apenas da topologia da rede e independem de sua marcação, portanto, são importantes para a análise dinâmica de um processo modelado. Estes correspondem aos conjuntos de lugares nos quais a soma de fichas permanece constante para todas as marcações alcançáveis pela rede. Dessa forma, a análise dos invariantes de lugar pode colaborar para a validação lógica de um modelo desenvolvido em RP. (Moody and Antsaklis, 1998) se fundamentam nessa definição para propor a síntese de supervisores em RP, tal que:

$$
\begin{gathered}
C_{c}=-\mathbf{L} C \\
M_{c_{0}}=b-\mathbf{L} M_{0}
\end{gathered}
$$

é a matriz de incidência do supervisor, $\mathbf{L}$ é a matriz de dimensão igual ao número de lugares da rede não controlada, em que cada um de seus elementos é igual a um, se o lugar for submetido a alguma restrição, sendo igual a zero, caso contrário; $C$ é a matriz de incidência da RP não controlada, $M_{c_{0}}$ é a marcação inicialdo supervisor, b é a matriz unitária cujo elemento é a quantidade de fichas impostas pela restrição ao sistema e M é a marcação inicial da rede não controlada.

Teorema 1 (Moody and Antsaklis, 1998) Dada a inequação

$$
b-\mathbf{L} M_{0} \geq 0
$$

então a $R P$ a ser controlada terá matriz de incidência do supervisor $C_{c}=-\mathbf{L} C$ e marcação inicial do supervisor dada por $M_{c_{0}}=b-\mathbf{L} M_{0}$.
Prova: De fato, se (9) não for verdade então $\mathbf{L} M_{0}>0$ e nesse caso, as condições iniciais da rede poderiam violar as restrições, o que seria um absurdo.

Por outro lado, se (9) é verdadeira, então existe $M_{c_{0}} \geq 0$, tal que,

$$
b-\mathbf{L} M_{0}-M_{c_{0}}=0 \Longrightarrow M_{c_{0}}=b-\mathbf{L} M_{0}
$$

ou seja, a condição inicial do controlador é definida por um vetor que representa a folga em cada uma das restrições representadas por $b \geq \mathbf{L} M_{0}$.

Além disso, a equação (7) força invariantes, incorrendo em um ciclo fechado da rede. Assim:

$$
\begin{aligned}
b & =\mathbf{L} M_{0}+M=\mathbf{L} M+M_{0} \Longrightarrow \\
b-\mathbf{L} M_{0} & =M=\mathbf{L} M+M_{0}-\mathbf{L} M_{0} \geq 0
\end{aligned}
$$

de acordo com (9), o que comprova o Teorema.

\subsection{O Método RCCD}

Segundo (Jensen and Kristensen, 2009), uma representação mais compacta de uma RPC é obtida através da associação, a cada ficha, de um conjunto de dados, denominados cores da ficha. O conceito de cor é análogo ao conceito de tipo, comum nas linguagens de programação. Dessa forma, as RPC são uma extensão às redes de Petri que combinam a estrutura de uma RP com uma linguagem de programação. Assim, pode haver diferenciação de fichas e associação de variáveis e funções aos arcos da rede em substituição aos pesos dos arcos das RP. Tal diferenciação de fichas se dá pelas cores assumidas por cada ficha, definidas previamente. A marcação de cada lugar de uma RPC é um subconjunto do conjunto de cores associado ao lugar. A utilização de subconjuntos é necessária para que um lugar possa armazenar duas ou mais fichas da mesma cor, o que caracteriza as múltiplas ocorrências de um mesmo elemento. Sendo assim, as RPC são capazes de modelar sistemas mais complexos de forma mais compacta e de fácil visualização.

Aproveitando-se das características das RPC, o método RCCD para a síntese de supervisores desenvolve- e a partir da análise da matriz de incidência da RPC, com a decomposição dessa matriz em outras matrizes de coefientes de uma mesma variável. Tal decomposição favorece o cálculo de supervisores podendo-se aplicar uma extensão do Teorema 1. Dessa forma é proposta uma solução para síntese de supervisores em RPC, utilizando a ferramenta CPNTools (Jensen and Kristensen, 2009). 
Seguindo o procedimento de decomposição das fichas coloridas, note-se que um lugar em uma RPC pode conter fichas de diferentes cores, as quais podem ou não sofrer restrições, independentemente. Nesse caso, pode-se definir dois subconjuntos de fichas, denominados:

- fichas de restrições, que são as fichas que estão submetidas a alguma restrição.

- fichas complementares de fluxo, que são as fichas do conjunto que não sofrem restrições.

Assim, dado um SED não controlado, a especificação de controle e seu modelo em RPC, a aplicação do método requer a definição da matriz $\mathbf{D}$ das funções associadas aos arcos para a separação de variáveis, segundo as cores. A matriz D, denominada matriz de incidência, é definida, tal que, os seus elementos são as funções ou variáveis associadas aos arcos da rede não controlada com os coeficientes de incidência. Didaticamente, consegue-se visualizar a decomposição de cores através da separação das variáveis associadas a elas. Após a decomposição de cores conservam-se, para efeito de análise, apenas os coeficientes que definem os lugares de entrada e saída das transições. Encontra-se assim, a matriz $\mathbf{C}$, matriz de referência de incidência, ou seja, se um elemento dessa matriz é igual a 1, isso indica que o lugar correspondente é saída de referida transição, se o elemento é igual a -1, então o lugar é entrada da transição e o lugar é igual a zero quando o lugar não é entrada nem saída da transição. A seguir, de posse da marcação inicial da rede não controlada e das restrições, aplicam-se os resultados do Teorema 2. Dessa forma, para se obter um supervisor seguindo o RCCD é necessário:

1. Construir a matriz de $\mathbf{D}$, denominada matriz de incidência da RPC não controlada, cujos elementos representam as respectivas expressões associadas aos arcos.

2. Decompor a matriz $\mathbf{D}$ em uma soma de matrizes onde cada parcela é uma matriz cujos elementos são relativos a uma mesma variável $x_{n}$, tal que, $\mathbf{D}=\mathbf{D}^{x_{1}}+\mathbf{D}^{x_{2}}+$ $\ldots+\mathbf{D}^{x_{n}}$.

3. Construir $\mathbf{C}$, denominada a matriz de referência de incidência da RPC não controlada, a partir da matriz $\mathbf{D}$, considerando-se apenas os coeficientes de incidência relativos às expressões associadas aos arcos.

4. Decompor a matriz $\mathbf{C}$ em uma soma de matrizes cujos elementos, coeficientes de incidência, estejam associados a uma mesma variável tomando como referencial as parcelas $\mathbf{D}^{x_{n}}$ de $\mathbf{D}$ decomposta, tal que, $\mathbf{C}=\mathbf{C}^{x_{1}}+\mathbf{C}^{x_{2}}+\ldots+\mathbf{C}^{x_{n}}$.
5. Para cada restrição imposta, adicionar o número de fichas correspondente à marcação inicial das outras cores (fichas complementares de fluxo), que são representadas pela mesma variável.

6. Calcular a(s) matriz(es) de incidência(s) e a(s) marcação(ões) iniciais do(s) controlador(es) usando uma extensão das equações (7) e (8) de Moody e Antsaklis, modificadas e adaptadas para uma RPC.

Seguindo a separação de variáveis pela decomposição da matriz $\mathbf{C}$, de referência de incidência da RPC, obtém-se a matriz $\mathbf{C}_{c}$, de incidência do supervisor, usando a equação (7). Salienta-se que, na matriz de incidência a ser decomposta, registra-se, não a quantidade de fichas, nem as transformações de fichas de determinadas cores por funções de arcos da rede, mas a incidência destas, independentes de seu formato. Observe-se que se as expressões de arcos da rede forem funções de uma mesma variável então não haverá necessidade da decomposição da matriz $\mathbf{C}$, bastando seguir os passos 2 e 3 do método. Sendo assim, tem-se o esquema para a matriz controlada $\mathbf{C}_{c}$, de um supervisor, em (12):

$$
\mathbf{C}_{c}=-\mathcal{L} \mathbf{C}=\left(\begin{array}{c}
\mathbf{L}_{1} \\
\mathbf{L}_{2} \\
\vdots \\
\mathbf{L}_{r}
\end{array}\right)\left(\begin{array}{ccc}
p_{1} t_{1} & \cdots & p_{1} t_{n} \\
\vdots & \ddots & \vdots \\
p_{m} t_{1} & \cdots & p_{m} t_{n}
\end{array}\right)
$$

em que $p_{i} t_{j}$ representa o peso do arco que liga $p_{i}$ a $t_{j}$. No RCCD pode-se trabalhar com $k$ cores e $r$ restrições para um dado modelo RPC. Nesse caso, a inequação (9) fica estendida em (13), tal que,

$$
(b+\hat{b})-\mathcal{L} \mathbf{M}_{0} \geq 0
$$

em que $\hat{b}$ é a matriz das fichas complementares de fluxo.

Dessa forma, tem-se que, existe $\mathbf{M}_{c_{0}} \geq 0$, tal que,

$$
\mathbf{M}_{c_{0}}=(b+\hat{b})-\mathcal{L} \mathbf{M}_{0}
$$

Daí, pode-se definir em (14) que:

- $b=\left(b_{i}\right)$ é a matriz $r \times 1$ das restrições impostas à rede e $b_{i}$ é a $i$-ésima restrição, tal que, $1 \leq i \leq r$. Se $b_{i}$ não é uma restrição então $b_{i}=0$. 


$$
b=\left(b_{i}\right)_{r \times 1}=\left(\begin{array}{c}
b_{1} \\
b_{2} \\
\vdots \\
b_{r}
\end{array}\right)
$$

- $\hat{b}=\left(\hat{b}_{i}\right)$ é a matriz $r \times 1$ dos conjuntos das fichas complementares de fluxo da rede relativas a $b_{i} \mathrm{e} \hat{b}_{i} \mathrm{o} i$-ésimo conjunto das fichas complementares de fluxo da rede (fichas de cores diferentes) relativas a $b_{i}$. Se $\hat{b}_{i}$ é relativa a $b_{i}=0$ então $\hat{b}_{i}=0$.

$$
\hat{b}=\left(\hat{b}_{i}\right)_{r \times 1}=\left(\begin{array}{c}
\hat{b}_{1} \\
\hat{b}_{2} \\
\vdots \\
\hat{b}_{r}
\end{array}\right)
$$

- $\mathcal{L}$ é a matriz composta por $r$ vetores $\left(\mathbf{L}_{1 \times m}\right)_{i}$ em que cada $\left(\mathbf{L}_{1 \times m}\right)_{i}=1$ define o(s) $m$-ésimo(s) lugar(es) relativo(s) à(s) $i$-ésima(s) restrição(ões). Caso $b_{i}=0$ então $\left(\mathbf{L}_{1 \times m}\right)_{i}=0$.

$$
\mathcal{L}=\left[\left(\mathbf{L}_{1 \times m}\right)_{i}\right]_{r \times m}=\left(\begin{array}{c}
\mathbf{L}_{1} \\
\mathbf{L}_{2} \\
\vdots \\
\mathbf{L}_{r}
\end{array}\right)
$$

- $\mathbf{M}_{0}$ é a marcação inicial da RPC.

$$
\mathbf{M}_{0}=\left[\mathbf{M}_{0}\left(p_{j}\right)\right]_{m \times 1}=\left(\begin{array}{c}
\mathbf{M}_{0}\left(p_{1}\right) \\
\mathbf{M}_{0}\left(p_{2}\right) \\
\vdots \\
\mathbf{M}_{0}\left(p_{m}\right)
\end{array}\right)
$$

- $\mathbf{M}_{c_{0}}$ é a marcação inicial do supervisor.

$$
\mathbf{M}_{c_{0}}=\left[\mathbf{M}_{c_{0_{i}}}\right]_{r \times 1}=\left(\begin{array}{c}
\mathbf{M}_{c_{0_{1}}} \\
\mathbf{M}_{c_{0_{2}}} \\
\vdots \\
\mathbf{M}_{c_{0_{r}}}
\end{array}\right)
$$

Segue que em (14) pode-se simplificar a expressão tomando $\mathbf{B}=b+\hat{b}$. Assim tem-se:

$$
\mathbf{M}_{c_{0}}=\mathbf{B}-\mathcal{L} \mathbf{M}_{0}
$$

marcação inicial do supervisor pelo método $R C C D$.

Dessa forma, a matriz $\mathbf{B}$, que apresenta as restrições $b_{i}$ somadas com as fichas complementares de fluxo $\hat{b}_{i}$, inerentes a cada uma delas, respectivamente, é dada por:

$$
\mathbf{B}=\left(\mathbf{B}_{i}\right)_{r \times 1}=\left(\begin{array}{c}
b_{1}+\hat{b}_{1} \\
b_{2}+\hat{b}_{2} \\
\vdots \\
b_{r}+\hat{b}_{r}
\end{array}\right)
$$

Note que cada $\hat{b}_{i}$ é um conjunto de fichas, complementar de $b_{i}$.

Assim:

$$
\mathbf{M}_{c_{0}}=\left(\begin{array}{c}
b_{1}+\hat{b}_{1} \\
b_{2}+\hat{b}_{2} \\
\vdots \\
b_{r}+\hat{b}_{r}
\end{array}\right)-\left(\begin{array}{c}
\mathbf{L}_{1} \\
\mathbf{L}_{2} \\
\vdots \\
\mathbf{L}_{r}
\end{array}\right)\left(\begin{array}{c}
\mathbf{M}_{c_{0_{1}}} \\
\mathbf{M}_{c_{0_{2}}} \\
\vdots \\
\mathbf{M}_{c_{0_{r}}}
\end{array}\right)
$$

Segue que, de (20):

$$
\mathbf{M}_{c_{0}}=\mathbf{B}-\mathcal{L} \mathbf{M}_{0} \Longleftrightarrow \mathbf{B}-\mathcal{L} \mathbf{M}_{0} \geq 0
$$

Tendo como hipótese inicial o Teorema 1 chega-se a uma fundamentação matemática da proposta baseada nos invariantes de lugar para o RCCD, exposta no Teorema 2.

\section{Teorema 2 Dada a expressão}

$$
\mathbf{B}-\mathcal{L} \mathbf{M}_{0} \geq 0
$$

consequente de (13), então a RPC a ser controlada terá marcação inicial do supervisor $\mathbf{M}_{c_{0}}$ dada por (20).

Prova: Por hipótese que a equação (9) é verdadeira. Tomando $\hat{b} \geq 0$ (o conjunto das fichas complementares de fluxo relativas a $b$ ) obtém-se (14), preservando a veracidade da expressão, o que implica em (24), onde tem-se $\mathbf{B} \geq \mathcal{L} \mathbf{M}_{0}$. Nesse caso, existe $\mathbf{M}_{c_{0}} \geq 0$, tal que,

$$
\mathbf{B}=\mathbf{M}_{c_{0}}+\mathcal{L} \mathbf{M}_{0}
$$


o que implica em (20).

Por outro lado, supondo por absurdo, que (24) é falsa, podese dizer que:

$$
\mathbf{B}<\mathcal{L} \mathrm{M}_{0}
$$

daí tem-se que,

$$
b+\hat{b}<\mathcal{L} \mathbf{M}_{0}
$$

o que implica,

$$
b<\mathcal{L} \mathbf{M}_{0}
$$

Como $\mathcal{L} \mathrm{M}_{0}>0$ tem-se que:

$$
b-\mathcal{L} \mathbf{M}_{0}<0
$$

um absurdo, pois contraria (9), logo, (23) é verdadeira, o que confirma o teorema.

\subsection{Contextos no RCCD}

A seguir são apresentados exemplos que são ilustrações de contextos onde se percebe a dinâmica do método. No exemplo 1, a RPC possui apenas um conjunto de cores (DATA) relativo a todos os lugares da rede e a todos os arcos é associada a variável $x$. Nesse caso, de variável única, $\mathbf{D}=\mathbf{D}^{x} \mathrm{e}$ $\mathbf{C}=\mathbf{C}^{x}$. O exemplo 2 mostra o método aplicado a uma rede com conjuntos distintos de cores e variáveis distintas associadas aos arcos, onde se ressalta o aparecimento de pesos diferentes de 1. Além disso, observa-se com propriedade que, as declarações nos arcos de controle na saída das transições, referente a cada variável, são um espelho das expressões associadas aos arcos de incidência dessas transições, segundo cada variável.

Exemplo 1 RPC na Figura 1, com um conjunto de cores onde uma única variável está associada aos arcos.

Impondo as especificações $\mathbf{M}\left(p_{2}\right) \leq 1^{\prime \prime} a_{1}$ " e $\mathbf{M}\left(p_{3}\right) \leq$ $1^{\prime \prime \prime} a_{2}$ " tem-se, da RPC não controlada, a matriz de incidência relativa à variável $x$

\section{DECLARATIONS}

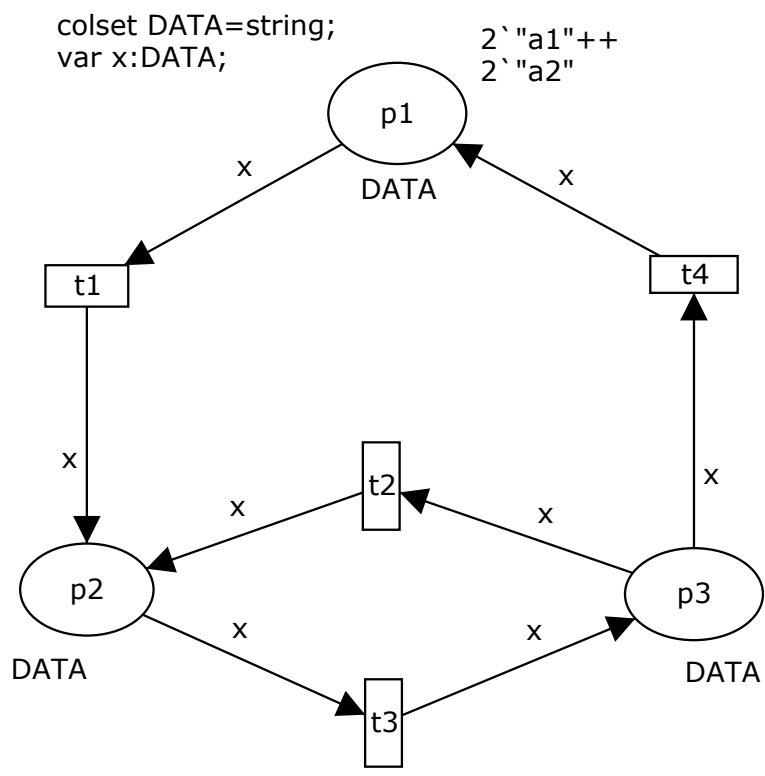

Figura 1: RPC não controlada onde os arcos estão associados a uma única variável.

$$
\mathbf{D}^{x}=\left(\begin{array}{cccc}
-1(x) & 0 & 0 & 1(x) \\
1(x) & 1(x) & -1(x) & 0 \\
0 & -1(x) & 1(x) & -1(x)
\end{array}\right)
$$

o que implica na matriz de referência de incidência, da RPC não controlada, relativa à variável $x$ em (31).

$$
\mathbf{C}^{x}=\left(\begin{array}{cccc}
-1 & 0 & 0 & 1 \\
1 & 1 & -1 & 0 \\
0 & -1 & 1 & -1
\end{array}\right)
$$

$\mathrm{O}$ vetor $\mathcal{L}$ que indica que os lugares que sofrem aes restritivas

$$
\mathcal{L}=\left(\begin{array}{lll}
0 & 1 & 0 \\
0 & 0 & 1
\end{array}\right)
$$

Assim, a matriz $\mathbf{C}_{c}$, do supervisor

$$
\mathbf{C}_{c}=-\mathcal{L} \mathbf{C}=\left(\begin{array}{cccc}
-1 & -1 & 1 & 0 \\
0 & 1 & -1 & 1
\end{array}\right)
$$




\section{DECLARATIONS}

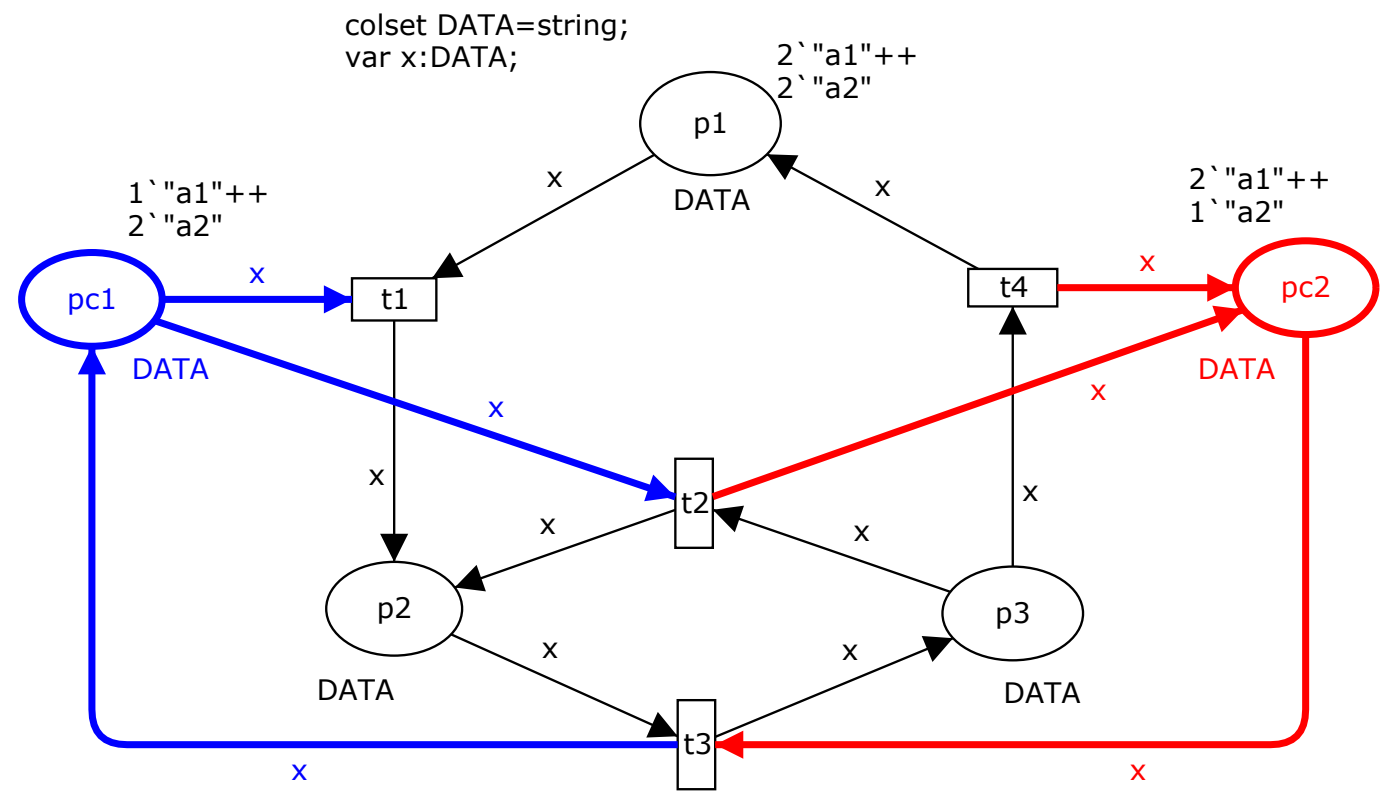

Figura 2: RPC controlada onde os comandos de arcos são representados por uma única variáel.

Dessa forma, o supervisor possui dois lugares de controle, $p c_{1}$ e $p c_{2}$, vistos na Figura 2, tal que, as linhas da matriz $\mathbf{C}_{c}$ so as matrizes de referncia de incidncias desse controladores.

A primeira linha, referente restrio $\mathbf{M}\left(p_{2}\right) \leq 1^{\prime \prime} " a_{1} "$ diz que o lugar de controle $p c_{1}$ ser entrada para as transies $t_{1}$ e $t_{2}$ e sada para $t_{3}$. A segunda linha, referente restrio $\mathbf{M}\left(p_{3}\right) \leq$ $1^{\prime \prime} " a_{2}$ ", diz que o lugar de controle $p c_{2}$ ser entrada de $t_{3} \mathrm{e}$ sada de $t_{2}$ e $t_{4}$. A seguir, as marcaes iniciais dos lugares de controle $p c_{1}$ e $p c_{2}$ so adquiridas em (36), segundo (22), sabendo-se que a matriz $\mathbf{B}$ que apresenta a soma das restries com as respectivas fichas complementares de fluxo é

$$
\mathbf{B}=\left(\begin{array}{l}
1^{\prime \prime \prime} a_{1} "+2^{\prime \prime \prime} a_{2} " \\
1^{\prime \prime \prime} a_{2} "+2^{\prime \prime \prime} a_{1} "
\end{array}\right)
$$

e a marcação inicial da RPC não controlada é

$$
\mathbf{M}_{0}=\left(\begin{array}{c}
2^{\prime \prime} a_{1} "+2^{\prime \prime \prime} a_{2} " \\
0 \\
0
\end{array}\right)
$$

Dessa forma,

$$
\begin{aligned}
\mathbf{M}_{c_{0}} & =\left(\begin{array}{l}
1^{\prime \prime \prime} a_{1} "+2^{\prime \prime \prime} a_{2} " \\
1^{\prime \prime \prime} a_{2} "+2^{\prime \prime \prime} a_{1} "
\end{array}\right) \\
& -\left(\begin{array}{lll}
0 & 1 & 0 \\
0 & 0 & 1
\end{array}\right)\left(\begin{array}{c}
2^{\prime \prime \prime} a_{1} "+2^{\prime \prime \prime} a_{2} " \\
0 \\
0
\end{array}\right) \\
& =\left(\begin{array}{l}
1^{\prime \prime \prime} a_{1} "+2^{\prime \prime \prime} a_{2} " \\
1^{\prime \prime \prime} a_{2} "+2^{\prime \prime \prime} a_{1} "
\end{array}\right) .
\end{aligned}
$$

As linhas da matriz $\mathbf{M}_{c_{0}}$ em (36) determinam as marcaes iniciais dos lugares de controle $p c_{1}$ e $p c_{2}$, na Figura 2, respectivamente.

Exemplo 2 Ilustração do método RCCD onde as expressões dos arcos são definidas por mais de uma variável na Figura 3.

A RPC em questão ilustra uma situação que reforça o método RCCD quando uma especificação é imposta ao lugar $p_{2}$ da rede, tal que, o conjunto de cores do tipo inteiro (INT) sofre modificações, por operações matemáticas, após o disparo de $t_{1}$ e $t_{2}$, ou seja, no disparo de $t_{1}$ é retirada uma ficha de valor 1 do lugar $p_{1}$ e acrescentada uma ficha de valor 3 ao lugar $p_{2}$. Com efeito, o problema considera a restrição em relação ao lugar $p_{2}$ como sendo: 


$$
\mathbf{M}\left(p_{2}\right) \leq 1^{\prime} 3 .
$$

Para o segundo conjunto de cores (DATA), representado pela variável $y$, considere a restrição ao lugar $p_{3}$, tal que,

$$
\mathbf{M}\left(p_{3}\right) \leq 1^{\prime \prime} a_{1} "
$$

Além disso, nesse exemplo, é apresentada uma expressão associada a um arco que está sujeita a condições do tipo IF. Nesse caso, para construção da matriz de incidência da RPC não controlada toma-se a união dos dados de saída que concluem essas condições, segundo a variável no contexto, processo elaborado seguindo as expressões (39) a (44).

Dessa forma, tem-se a matriz $\mathbf{D}$, referente à RPC não controlada:

$\mathbf{D}=\left(\begin{array}{cccc}-1(x) & 0 & 0 & 1(x) \\ 1(3 x, y) & 1(3 x, y) & -1(x, y) & 0 \\ 0 & -1(x, y) & 1[(1, y) \mathrm{v}(2, y)] & -1(x, y) \\ -1(y) & 0 & 0 & 1(y)\end{array}\right)$

Separando-se as variáveis obtém-se:

$$
\mathbf{D}=\mathbf{D}^{x}+\mathbf{D}^{y}
$$

em que a matriz de incidência relativa à variável $x$ :

$$
\mathbf{D}^{x}=\left(\begin{array}{cccc}
-1(x) & 0 & 0 & 1(x) \\
1(3 x) & 1(3 x) & -1(x) & 0 \\
0 & -1(x) & 1[1 \mathrm{v} 2] & -1(x) \\
0 & 0 & 0 & 0
\end{array}\right)
$$

o que implica na matriz de referência de incidência relativa à variável $x$

$$
\mathbf{C}^{x}=\left(\begin{array}{cccc}
-1 & 0 & 0 & 1 \\
1 & 1 & -1 & 0 \\
0 & -1 & 1[1 \mathrm{v} 2] & -1 \\
0 & 0 & 0 & 0
\end{array}\right)
$$

A matriz de incidência para a variável $y$

$$
\mathbf{D}^{y}=\left(\begin{array}{cccc}
0 & 0 & 0 & 0 \\
1(y) & 1(y) & -1(y) & 0 \\
0 & -1(y) & 1[y \mathrm{v} y] & -1(y) \\
-1(y) & 0 & 0 & 1(y)
\end{array}\right)
$$

o que implica na matriz de referência de incidência, sob influência da variável $y$, da RPC não controlada

$$
\mathbf{C}^{y}=\left(\begin{array}{cccc}
0 & 0 & 0 & 0 \\
1 & 1 & -1 & 0 \\
0 & -1 & 1 & -1 \\
-1 & 0 & 0 & 1
\end{array}\right)
$$

De acordo com as especificações em (37) e (38) tem-se:

$$
\mathcal{L}=\left(\begin{array}{c}
\mathbf{L}_{1} \\
\mathbf{L}_{2}
\end{array}\right)=\left(\begin{array}{llll}
0 & 1 & 0 & 0 \\
0 & 0 & 1 & 0
\end{array}\right)
$$

donde a matriz do supervisor é:

$$
\mathbf{C}_{c}=-\left(\begin{array}{c}
\mathbf{L}_{1} \mathbf{C}^{x} \\
\mathbf{L}_{2} \mathbf{C}^{y}
\end{array}\right)
$$

tal que,

$$
\mathbf{C}_{c}=\left(\begin{array}{cccc}
-1 & -1 & 1 & 0 \\
0 & 1 & -1 & 1
\end{array}\right)
$$

donde se definem os lugares de controle $p c_{3}$ e $p c_{4}$, vistos na Figura 4.

Além disso, tem-se a marcação inicial da rede

$$
\mathbf{M}_{0}=\left(\begin{array}{c}
2^{\prime} 1++2^{\prime} 2 \\
0 \\
0 \\
2^{\prime \prime \prime} a_{1} "++2^{\prime \prime \prime} a_{2} "
\end{array}\right)
$$

e a matriz $\mathbf{B}$ com as restrições impostas à rede e as relativas fichas complementares de fluxo, tal que,

$$
\mathbf{B}=\left(\begin{array}{c}
1^{\prime} 1++2^{\prime} 2 \\
1^{\prime \prime \prime} a_{1}^{\prime \prime}++2^{\prime \prime \prime} a_{2} "
\end{array}\right)
$$






Figura 3: RPC não controlada sob influência de duas variáveis.

Dessa forma, a marcação inicial do supervisor associado aos lugares $p c_{3}$ e $p c_{4}$, na Figura 4, é:

$$
\begin{aligned}
\mathbf{M}_{c_{0}} & =\left(\begin{array}{c}
1^{\prime} 1++2^{\prime} 2 \\
1^{\prime \prime \prime} a_{1} "++2^{\prime \prime \prime} a_{2} "
\end{array}\right) \\
& -\left(\begin{array}{cccc}
0 & 1 & 0 & 0 \\
0 & 0 & 1 & 0
\end{array}\right)\left(\begin{array}{c}
2^{\prime} 1++2^{\prime} 2 \\
0 \\
0 \\
2^{\prime \prime \prime} a_{1} "++2^{\prime \prime} a_{2} "
\end{array}\right) \\
& =\left(\begin{array}{c}
1^{\prime} 1++2^{\prime} 2 \\
1^{\prime \prime \prime} a_{1} "++2^{\prime \prime \prime} a_{2} "
\end{array}\right) .
\end{aligned}
$$

ELSE, as expressões associadas a este arco são do mesmo tipo, ou seja, expressões com IF-THEN-ELSE, relacionadas à variável $x$ do contexto. Esse fato reforça a importância da matriz de incidência no RCCD quando na decomposição das cores através da separação das variáveis.

\section{FUSÃO DE CONTROLADORES}

Nesta seção, é mostrado o método para definição de supervisores resultantes da fusão de controladores. O método é uma extensão do Teorema 2, que comprova o RCCD, mostrado no Teorema 3. A seguir são apresentados exemplos como ilustração do método.

Note que na definição do arco que incide no controlador $p c_{3}$, na Figura 4, o qual envolve as condições do tipo IF-THEN- 


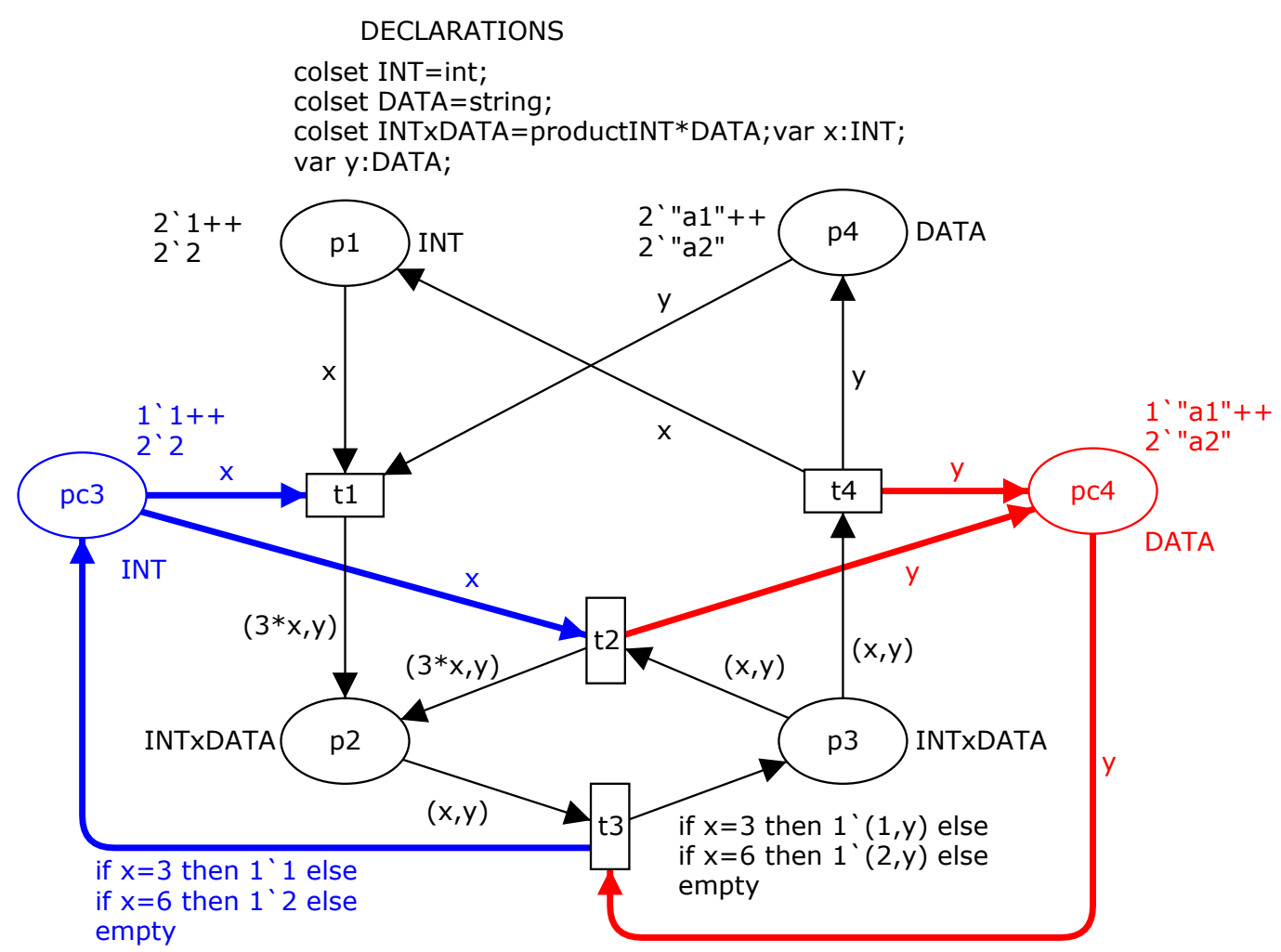

Figura 4: RPC controlada sob influência de duas variáveis.

No caso em que o RCCD é aplicado em uma rede que apresente diferentes conjuntos de cores associados a diferentes lugares e expressões de arcos compostas por variáveis distintas, na Figura 5, pode-se definir as especificações como um só conjunto, sem nenhum prejuízo para o sistema modelado, o que leva à fusão dos controladores. Este fato é devido à identificação das cores distintas no processo de separação das variáveis, na decomposição da matriz de incidência. Dessa forma, a matriz $\mathcal{L}$ determina controladores relativos aos conjuntos de cores, distintamente. Assim, na fusão desses controladores, o controlador resultante agrega toda ação de incidência sobre as transições inerentes às especificações dadas, podendo-se impor arcos de incidência a uma mesma transição, de entrada e/ou saída, sob ação de variáveis distintas. Nessas condições o supervisor é não-bloqueante. Esse processo é mostrado no Teorema 3 , onde se define a marcação inicial de fusão $\mathcal{M}_{0}$ e a matriz de incidência de fusão $\mathcal{H}$, do controlador resultante da fusão, o qual passa a exercer a função de supervisão total do sistema.

Teorema 3 Dada uma RPC com conjuntos de cores representados por variáveis distintas, sejam $\mathbf{B}_{i}$ e $\mathbf{M}_{0}$, a matriz das restrições com as relativas fichas complementares de fluxo e a marcação inicial da RPC controlada, então a marcação inicial e a matriz de incidência de fusão do supervisor são, respectivamente,

$$
\begin{gathered}
\mathcal{M}_{0}=\sum_{i=1}^{r} \mathbf{B}_{i}+\mathcal{L}_{i} \mathbf{M}_{0}, \\
\mathcal{H}=-\left(\sum_{i=1}^{r} \mathcal{L}_{i}\right) \mathbf{C} .
\end{gathered}
$$

Prova: Sejam $b$ e $\mathbf{M}_{0}$, respectivamente admissíveis, então

$$
\mathcal{L} \mathbf{M}_{0} \leq b \Longrightarrow \mathcal{L} \mathbf{M}_{0} \leq b+\hat{b} \Longrightarrow \mathcal{L} \mathbf{M}_{0} \leq \mathbf{B}
$$

Segundo o Teorema 1, sem perda de generalidade, pode-se considerar que

$$
\begin{aligned}
\mathcal{L}_{1} \mathbf{M}_{0}+\mathbf{M}_{c} & =\mathbf{B}_{1}, \\
\mathcal{L}_{2} \mathbf{M}_{0}+\mathbf{M}^{\prime}{ }_{c} & =\mathbf{B}_{2} .
\end{aligned}
$$


Com efeito, somando (54) e (55) tem-se

$$
\left(\mathcal{L}_{1}+\mathcal{L}_{2}\right) \mathbf{M}_{0}+\left(\mathbf{M}_{c}+\mathbf{M}^{\prime}{ }_{c}\right)=\mathbf{B}_{1}+\mathbf{B}_{2}
$$

o que implica,

$$
\mathcal{H}_{0}=\mathbf{M}_{c}+\mathbf{M}^{\prime}{ }_{c}=\left(\mathbf{B}_{1}+\mathbf{B}_{2}\right)-\left(\mathcal{L}_{1}+\mathcal{L}_{2}\right) \mathbf{M}_{0}
$$

Generalizando, $i=1: r$, tm-se (51).

Alm disso, seja,

$$
\mathbf{C}_{c}=-\left(\begin{array}{c}
\mathcal{L}_{1} \\
\mathcal{L}_{2}
\end{array}\right) \mathbf{C}
$$

a matriz de incidência dos supervisores em hipótese. Como, no contexto, a rede possui variáveis distintas para representar os diferentes conjuntos de cores então cada coordenada nos $\mathcal{L}_{i}$ são identificadas distintamente. Dessa forma, podese somar os $\mathcal{L}_{i}$ definindo supervisores sem conflito. Assim, a matriz de incidência de fusão dos supervisores é dada por

$$
\mathcal{H}=-\left(\mathcal{L}_{1}+\mathcal{L}_{2}\right) \mathbf{C}
$$

Com generalidade, para $i=1: r$ obtm-se (52), o que comprova o teorema.

Exemplo 3 Considere a RPC com dois conjuntos de cores distintos, onde um mesmo conjunto de cores é representado por duas variáveis na Figura 5.

Nesse contexto, a RPC é submetida a ação do supervisor definido pelos lugares de controle $p c_{5}$ e $p c_{6}$, na Figura 6. Seguindo o Teorema 3 é feita a Fusão dos Controladores definindo o lugar de controle $p c r$, Figura 7, o qual assume superviso total requerida.

Considere as especificaes:

$$
\begin{aligned}
& \mathbf{M}\left(p_{3}\right)+\mathbf{M}\left(p_{4}\right) \leq 1^{\prime} 1, \\
& \mathbf{M}\left(p_{3}\right)+\mathbf{M}\left(p_{6}\right) \leq 2^{\prime} 2 .
\end{aligned}
$$

Nesse caso, da RPC não controlada na Figura 5, tem-se a matriz de incidência $\mathbf{D}$ em (62), de onde as matrizes de incidência de referência em relação às variáveis $x$ e $y$ são obtidas pela decomposição de $\mathbf{D}$ respectivamente, em (63) e (64).

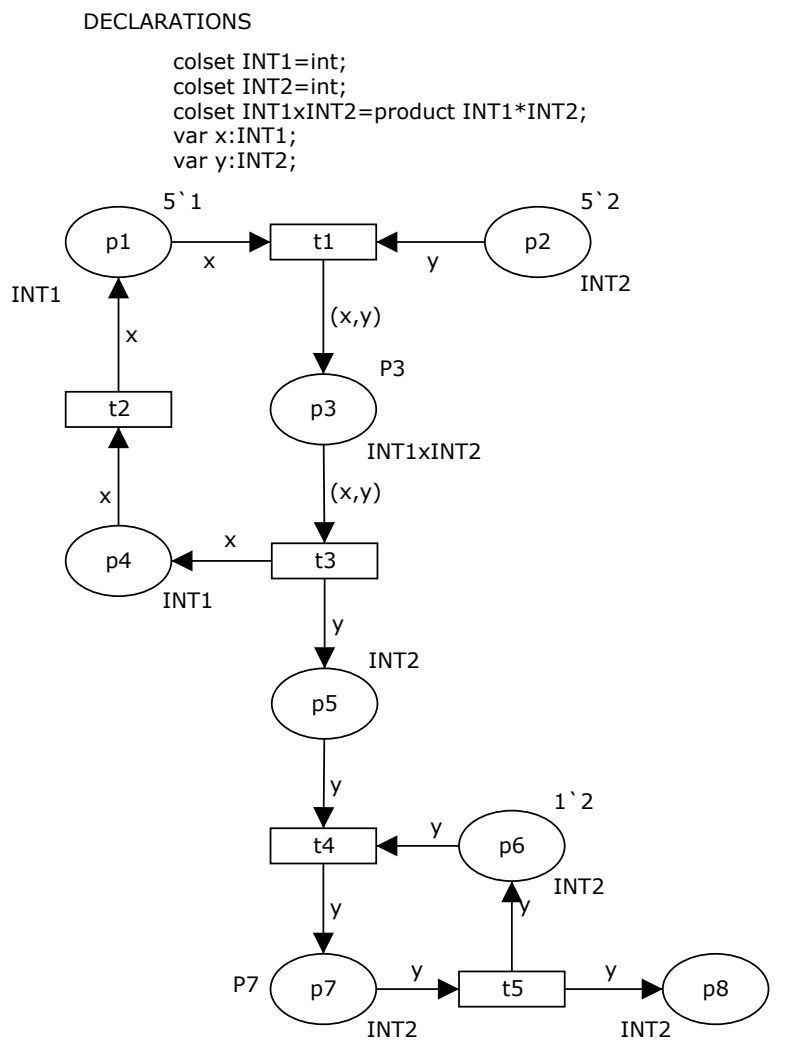

Figura 5: RPC não controlada com conjuntos de cores diferentes com variáveis distintas associadas aos arcos.

$\mathbf{D}=\left(\begin{array}{ccccc}-1(x) & 1(x) & 0 & 0 & 0 \\ -1(y) & 0 & 0 & 0 & 0 \\ 1(x, y) & 0 & -1(x, y) & 0 & 0 \\ 0 & -1(x) & 1(x) & 0 & 0 \\ 0 & 0 & 1(y) & -1(y) & 0 \\ 0 & 0 & 0 & -1(y) & 1(y) \\ 0 & 0 & 0 & 1(y) & -1(y) \\ 0 & 0 & 0 & 0 & 1(y)\end{array}\right)$

$$
\mathbf{C}^{x}=\left(\begin{array}{ccccc}
-1 & 1 & 0 & 0 & 0 \\
0 & 0 & 0 & 0 & 0 \\
1 & 0 & -1(x, y) & 0 & 0 \\
0 & -1 & 1 & 0 & 0 \\
0 & 0 & 0 & 0 & 0 \\
0 & 0 & 0 & 0 & 0 \\
0 & 0 & 0 & 0 & 0 \\
0 & 0 & 0 & 0 & 0
\end{array}\right)
$$




$$
\mathbf{C}^{y}=\left(\begin{array}{ccccc}
0 & 0 & 0 & 0 & 0 \\
-1 & 0 & 0 & 0 & 0 \\
1 & 0 & -1 & 0 & 0 \\
0 & 0 & 0 & 0 & 0 \\
0 & 0 & 1 & -1 & 0 \\
0 & 0 & 0 & -1 & 1 \\
0 & 0 & 0 & 1 & -1 \\
0 & 0 & 0 & 0 & 1
\end{array}\right)
$$

Como a marcação inicial da RPC não controlada é

$$
\mathbf{M}_{0}=\left(\begin{array}{c}
5^{\prime} 1 \\
5^{\prime} 2 \\
0 \\
0 \\
0 \\
1^{\prime} 2 \\
0 \\
0
\end{array}\right)
$$

e a matriz indicadora dos lugares que sofrem restrições é

$$
\mathcal{L}=\left(\begin{array}{llllllll}
0 & 0 & 1 & 1 & 0 & 0 & 0 & 0 \\
0 & 0 & 1 & 0 & 0 & 1 & 0 & 0
\end{array}\right)
$$

a matriz de incidência do supervisor desejado é

$$
\mathbf{C}_{c}=\mathcal{L} \mathbf{C}=\left(\begin{array}{ccccc}
-1 & 1 & 0 & 0 & 0 \\
-1 & 0 & 1 & 1 & -1
\end{array}\right)
$$

No contexto, tem-se que:

$$
\mathbf{B}=\left(\begin{array}{c}
1^{\prime} 1+0 \\
2^{\prime} 2+0
\end{array}\right)
$$

Note-se que não existem fichas complementares de fluxo relacionadas às restrições impostas. Assim, as marcações iniciais dos lugares de controle $p c_{5}$ e $p c_{6}$, na Figura 6, so obtidas em (69):

$$
\mathbf{M}_{c_{0}}=\mathbf{B}-\mathcal{L} \mathbf{M}_{0}=\left(\begin{array}{c}
1^{\prime} 1 \\
2^{\prime} 2
\end{array}\right)-\left(\begin{array}{c}
0 \\
1^{\prime} 2
\end{array}\right)=\left(\begin{array}{c}
1^{\prime} 1 \\
1^{\prime} 2
\end{array}\right)
$$

Para definir o supervisor resultante, definido pelo lugar de controle $p c r$, da Figura 7, correspondente à fusão dos lugares de controle $p c_{5}$ e $p c_{6}$ na Figura 6, deve-se estabelecer o conjunto de restries nos lugares $\mathrm{j}$ determinados, tal que,

$$
\mathbf{M}\left(p_{3}\right)+\mathbf{M}\left(p_{4}\right)+\mathbf{M}\left(p_{6}\right) \leq 1^{\prime} 1+2^{\prime} 2
$$

Note-se que em (70) não se repete o lugar $p_{3}$ que faz parte de (60) e (61). Isto ocorrendo, seria como se tivéssemos um lugar a mais na rede que traria uma repetição de incidência sobre a transição relativa à especificação imposta. Nesse caso, elimina-se a ação conflitante dos controladores transformando as especificações (60) e (61) numa especificação única (70).

Seguindo o Teorema 3, tem-se a matriz de incidência de fusão $\mathcal{H}$ que define o supercisor $p c r$, fruto da fusão dos controladores associados aos lugares $p c_{5}$ e $p c_{6}$, tal que:

$$
\mathcal{H}=\left(\begin{array}{lllll}
-2 & 1 & 1 & 1 & -1
\end{array}\right) .
$$

A marcação inicial de fusão $\mathcal{M}_{0}$, calculada a partir de (51) é dada por (72).

Pela equação (71) pode-se notar que o supervisor $p c r$, resultante de fusão, será entrada para a transição $t_{1}$ duas vezes, obviamente $\operatorname{com} x$ e com $y$, ser entrada para a transio $t_{5}$ e ser sada para as transies $t_{2}, t_{3}$ e $t_{4}$. O clculo em (72)

$$
\begin{aligned}
\mathcal{M}_{0}=\left[\left(1^{\prime} 1+0\right)+\left(2^{\prime} 2+0\right)\right] & \\
- & \left(\begin{array}{llllllll}
0 & 0 & 2 & 1 & 0 & 1 & 0 & 0
\end{array}\right)\left(\begin{array}{c}
5^{\prime} 1 \\
5^{\prime} 2 \\
0 \\
0 \\
0 \\
1^{\prime} 2 \\
0 \\
0
\end{array}\right) \\
& =\left(1^{\prime} 1+1^{\prime} 2\right) .
\end{aligned}
$$

mostra que a marcao inicial em $p c r$ ter uma ficha da cor $1 \mathrm{e}$ duas fichas da cor 2.

Dessa forma, o lugar $p c r$ resultante da fuso na Figura 7 agora o lugar de controle que assume a ao dos controladores representados pelos lugares $p c_{5}$ e $p c_{6}$ na Figura 6, simultaneamente. 


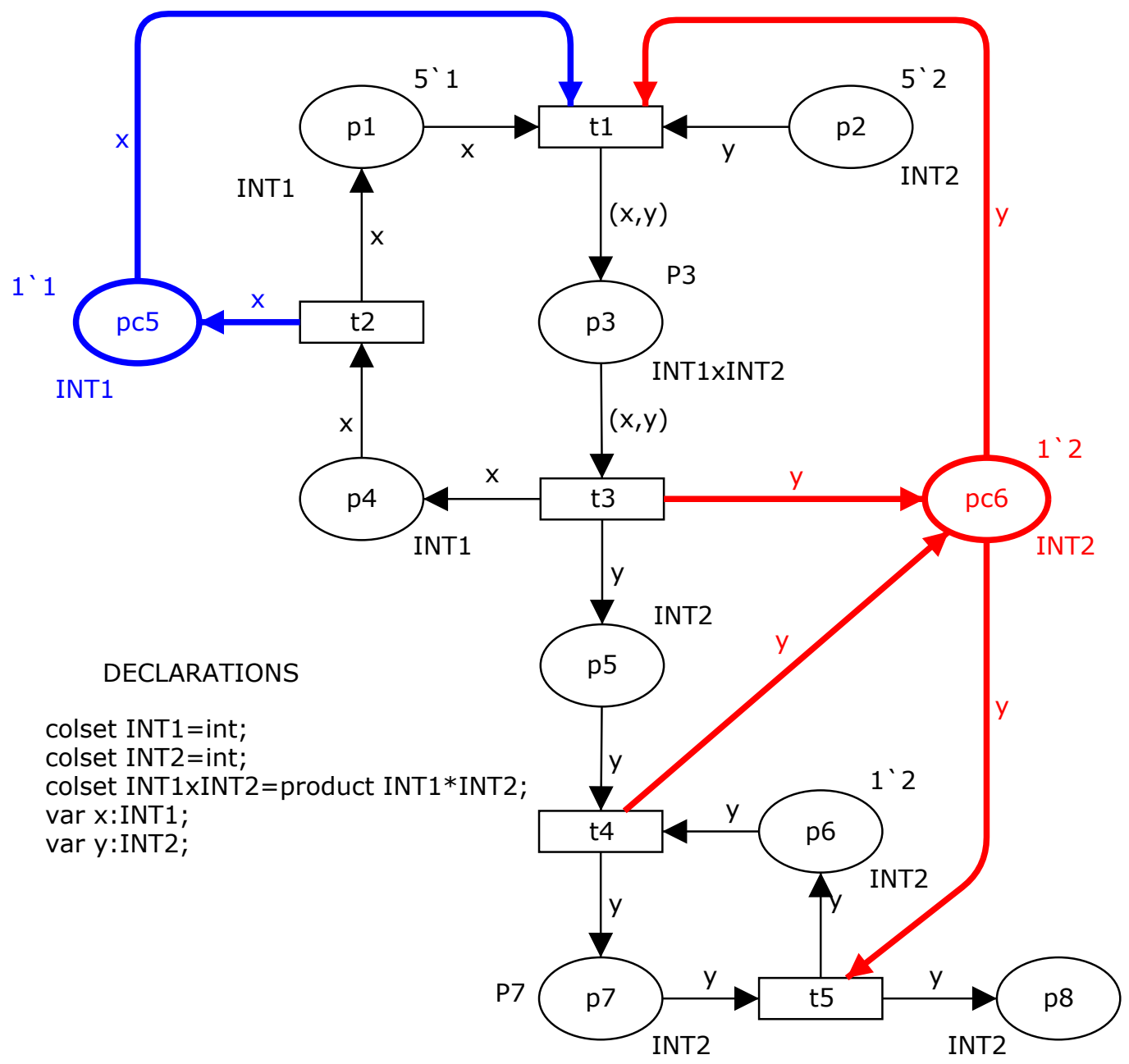

Figura 6: Rede controlada com cores do mesmo tipo representadas por variáveis distintas.

\subsection{Particularidade do Método de Fusão}

Considere a RPC apresentada na Figura 1, controlada pelo supervisor resultante de fuso pcr $_{1}$ na Figura 8.

Nesse exemplo as cores são associadas a uma mesma variá-

$$
\mathcal{M}_{0}=\left(1^{\prime \prime} a_{1} "+1^{\prime \prime \prime} a_{2} "\right) \text {. }
$$
vel, impossibilitando a decomposição da matriz de incidência e, portanto, obtendo-se um controle mais restritivo sobre as fichas complementares de fluxo. Dessa forma, quando se tem arcos de entrada e saída para uma mesma transição, eles se anulam na matriz de incidência.

Para definio do $p c r_{1}$, seguindo o Teorema 3 e dados do Exemplo 1, tem-se a matriz de incidncia de fuso.

$$
\mathcal{H}=\left(\begin{array}{llll}
-1 & 0 & 0 & 1
\end{array}\right)
$$

e a marcao inicial de fuso

Nesse caso, o supervisor resultante de fusão pcr 1 passa a exercer uma restrição maior ao sistema, impedindo a circulação das fichas complementares de fluxo, a fim de evitar o conflito provocado pela representação das diferentes cores representadas por uma mesma variável e o cumprimento das especificações desejadas. Assim, é necessário ponderar a aplicação da fusão de controladores nesse contexto, considerando custos e benefícios dessa perda, causada pela restrição ao movimento das fichas complementares de fluxo, para o sistema modelado. 


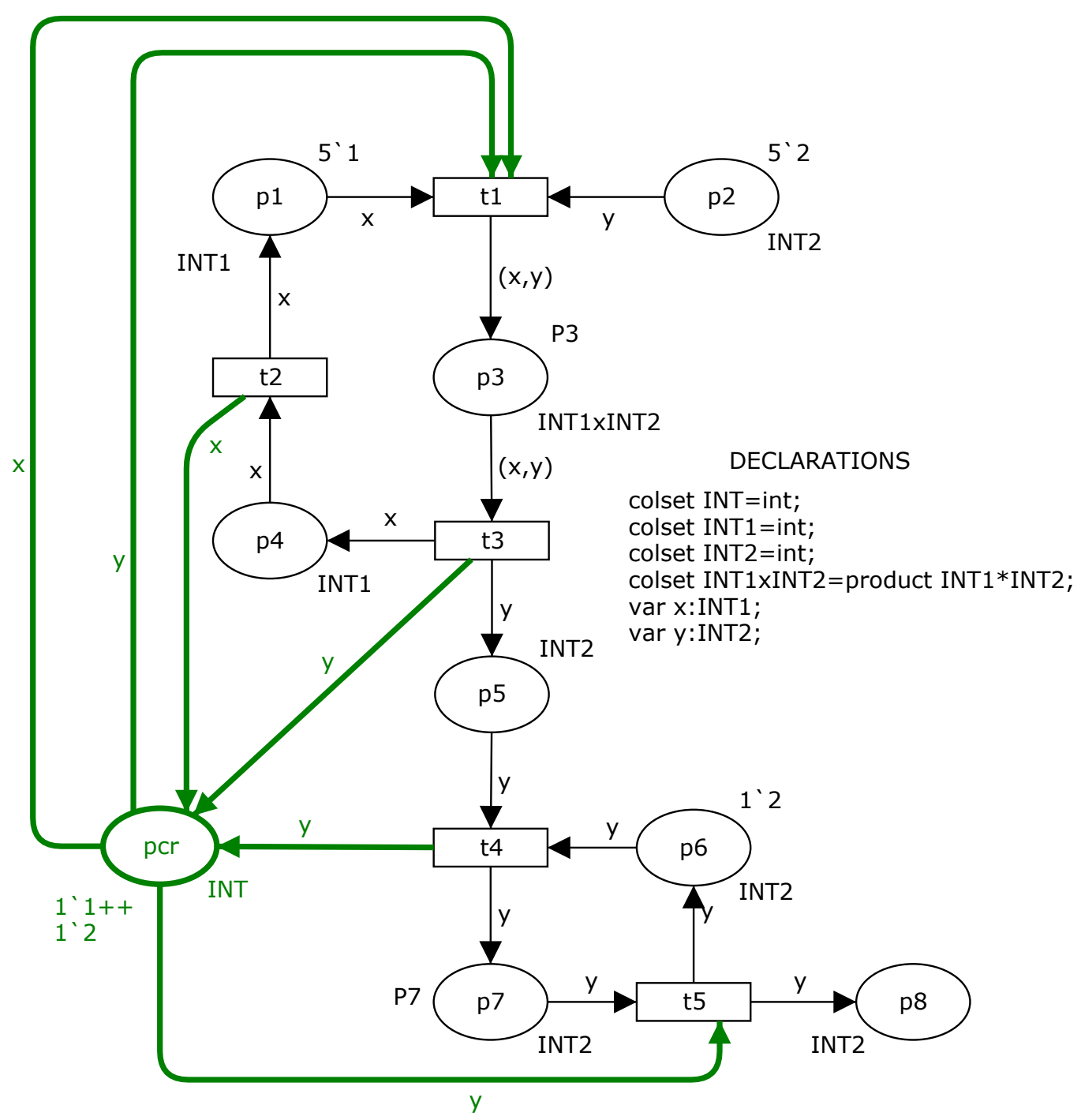

Figura 7: Rede controlada pelo supervisor $p c r$ resultante de fuso.

\section{CONCLUSÃo}

Neste trabalho é apresentada uma extensão do Teorema 1, de Moody and Antsaklis (1998), que é uma proposta para síntese de supervisores utilizando redes de Petri (RP). Seguindo a fundamentação matemática desse teorema, propõe-se a ampliação da proposta inicial em RP, para redes de Petri coloridas (RPC), apresentando o método de cálculo para supervisores em RPC denominado Restrições de Controle sobre Cores Decompostas - RCCD, explorando a decomposição da matriz de incidência da RPC a fim de definir supervisores que não sejam conflitantes em suas ações, nos contextos abordados, utilizando a ferramenta CPNTools.

Além disso, tomando como ponto de fundamentação teórica resultados sobre os invariantes de lugar de (Moody and
Antsaklis, 1998), é proposto o conceito de fusão dos lugares de controle desses supervisores em RPC, obtendo mesma performance do sistema, ou seja, tanto o supervisor original quanto o supervisor resultante da fusão oferecem mesma ação restritiva, segundo critérios formalizados no Teorema 3 e contextualizados. Uma particularidade do método para fusão de controladores ocorre quando há representação de cores diferentes por uma mesma variável da RPC não controlada. Isso impossibilita a decomposição da matriz de incidência, obtendo-se um controle mais restritivo sobre as fichas complementares de fluxo. Nesse caso, para que não aconteça conflito na ação do supervisor gerado pela fusão, as fichas complementares de fluxo são desprezadas nesse processo buscando o funcionamento do sistema com as especificações impostas, dentro de uma ação mais restritiva para o mesmo. Dessa forma, os métodos apresentados atendem aos 


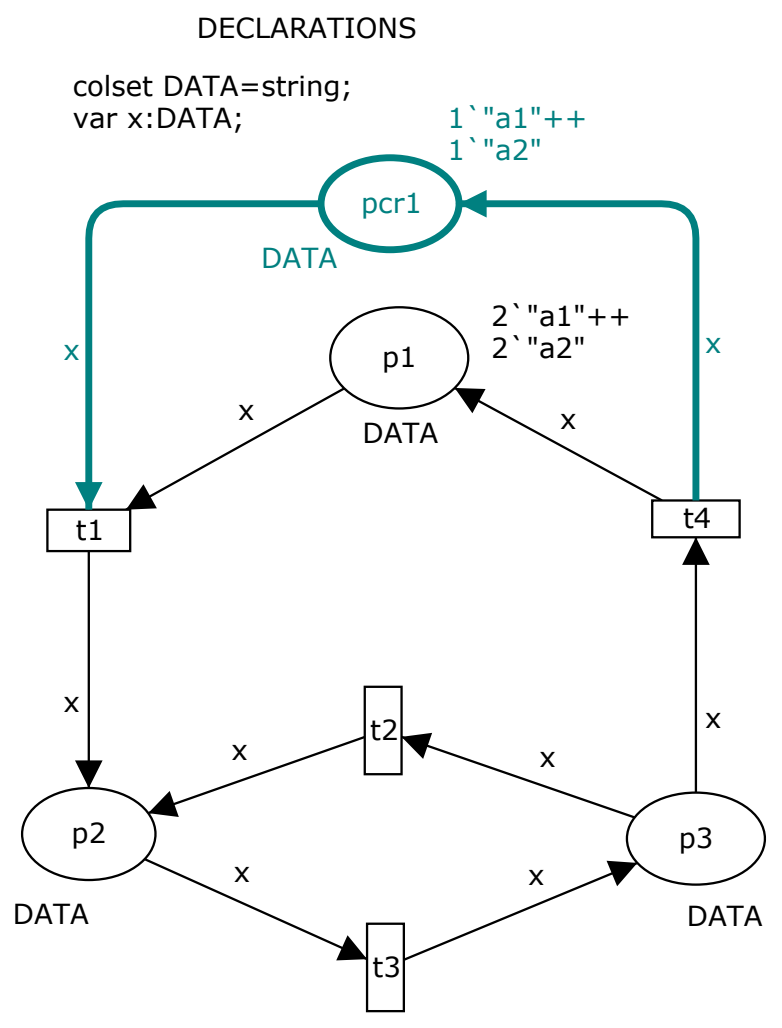

Figura 8: RPC controlada pelo supervisor pcr $_{1}$ resultante de fuso dos lugares de controle $p c_{1}$ e $p c_{2}$ na Figura 2.

conceitos da TCS em abordagens teóricas específicas inerentes às RPC, as quais podem modelar sistemas reais mais complexos.

Como trabalho futuro, propõe-se a elaboração de uma teoria sistemática para a fusão de controladores, em supervisores modelados por RPC, baseada na teoria de controle supervisório e a aplicação do método RCCD com a fusão de controladores em células de manufatura, atualmente em estudo.

\section{REFERÊNCIAS}

Barkaoui, K., Chaoui, A. and Zouari, B. (1997). Supervisory control of discrete event systems based on structure theory of petri nets, IEEE International Conference on Systems, Man, and Cybernetics, 1997. Computational Cybernetics and Simulation., Vol. 4, pp. 3750-3755.

Barroso, G. C. (1996). Uma Nova Abordagem para Snteses de Supervisores a Eventos Discretos, $\mathrm{PhD}$ thesis, Universidade Federal da Paraba.

Barroso, G. C., Lima, A. and Perkusich (1996). Synthesis of supervisors using petri nets, Proceedings of the 13th World Congress of IFAC (International Federation of
Automatic Control), Vol. J, San Franscisco, CA, USA, pp. $449-454$.

Braga, R. (2006). Contribuies ao controle supervisrio de sistemas a eventos discretos modelados por redes de pedtri, Master's thesis, Universidade Federal de Santa Catarina.

Cassandras, C. and Lafortune, S. (2008). Introduction to Discrete Event Systems (The International Series on Discrete Event Dynamic Systems), 2 edn, Springer.

Cury, J. (2001). Teoria de controle supervisrio de sistemas a eventos discretos.

Giua, A. (1992). Petri net as discrete event models for supervisory control, $\mathrm{PhD}$ thesis, Rensselaer Polytechnic Institute, New York.

Holloway, L. E., Krogh, B. H. and Giua, A. (1997). A survey of petri net methods for controlled discrete event systems, Discrete Event Dynamic Systems 7(2): 151-190.

Holloway, L. and Krogh, B. (1990). Synthesis of feedback control logic for a class of controlled petri nets, IEEE Transactions on Automatic Control 35(5): 514-523.

Hopcroft, J., Motwani, R. and Ullman, J. (2006). Introduction to Automata Theory Languages and Computation, 3 edn, Addison Wesley.

Iordache, M. V. and Antsaklis, P. J. (2006). Supervisory Control of Concurrent Systems: A Petri Nets Structural Approach, Boston, Birkhauser.

Jensen, K. and Kristensen, L. M. (2009). Coloured Petri Nets: Modelling and Validation of Concurrent Systems, 1 edn, Springer.

Lima, E. A. and Oliveira, P. J. (2007). Projeto de anlise e controle supervisrio de sistemas a eventos discretos usando redes de petri temporais, Technical report, Centro de Pesquisa e tecnologia da Universidade do Estado da Bahia, Salvador.

Makungu, M., Barbeau, M. and St-Denis, R. (1999). Synthesis of controllers of processes modeled as colored petri nets, Discrete Event Dynamic Systems 9: 147-169.

Moody, J. and Antsaklis, P. (1998). Supervisory Control of Discrete Event Systems Using Petri net, Kluwer Academic Publishers.

Moody, J., Antsaklis, P. and Lemmon, M. (1996). Petri net feedback controller design for a manufacturing system, 3th World Congress of IFAC, Vol. B, Discrete Event Models in Manufacturing, pp. 67-72. 
Murata, T. (1989). Petri nets: Properties, analysis and applications, Proceedings of the IEEE 77(4): 541-580.

Prata, B. A., Barroso, G. C. and Arruda, J. B. F. (2008). Um novo mtodo para controle supervisrio de sistemas a eventos discretos baseado em redes de petri coloridas, XV Simpsio Brasileiro de Pesquisa Operacional.

Ramadge, P. J. and Wonham, W. M. (1987). Supervisory control of a class of discrete event processes, SIAM Journal on Control and Optimization 25(1): 206-230.

Ramadge, P. and Wonham, W. (1989). The control of discrete event systems, Proceedings of the IEEE 77(1): 81-98. 